\title{
Yoga therapy to reduce fatigue in cancer: effects of reminder e-mails and long-term efficacy
}

\author{
Teresa Zetzl ${ }^{1}$ (1) $\cdot$ Andre Pittig ${ }^{2,3} \cdot$ Agnes Renner $^{1} \cdot$ Birgitt van Oorschot ${ }^{1} \cdot$ Elisabeth Jentschke ${ }^{1,4}$
}

Received: 11 November 2020 / Accepted: 6 June 2021 / Published online: 22 June 2021

(c) The Author(s) 2021

\begin{abstract}
Objective To examine the efficacy of reminder e-mails to continue yoga therapy on practice frequency and fatigue in cancer patients and long-term effects of yoga on fatigue, depression, and quality of life.

Methodology One hundred two cancer patients who completed an 8-week yoga therapy were randomly allocated to two groups: reminder $(N=51)$ vs. no-reminder group $(N=51)$. After completing yoga therapy, the reminder group received weekly e-mails for 24 weeks, which reminded them of practicing yoga, whereas the no-reminder group did not. Primary outcomes were fatigue and practice frequency, and long-term outcomes were fatigue, depression, and quality of life. Data were assessed using questionnaires after yoga therapy (T1) and 6 months after completing yoga therapy (T2).

Result A significantly stronger reduction of general $(p=0.038, d=0.42)$ and emotional fatigue $(p=0.004, d=0.59)$ and a higher increase of practice frequency $(p=0.015, d=0.52)$ between T1 and T2 were found for the reminder group compared to the no-reminder group. In the mediation model, practice frequency as a mediator partially explained the changes in emotional fatigue (indirect effect $B=-0.10$ ). Long-term effects of yoga therapy regarding fatigue, depression, and quality of life were found $(F>7.46, p<0.001, d>0.54)$.

Conclusion Weekly reminder e-mails after yoga therapy can positively affect general and emotional fatigue and help cancer patients with fatigue establish a regular yoga practice at home. However, higher practice frequency did not lead to higher physical or cognitive fatigue improvement, suggesting other factors that mediate efficacy on physical or cognitive fatigue, such as mindfulness or side effects of therapy.
\end{abstract}

Keywords Mind-body intervention $\cdot$ Complementary alternative medicine $\cdot$ Long-term effects $\cdot$ Yoga $\cdot$ Fatigue $\cdot$ Reminder e-mails

Teresa Zetzl

Zetzl_T@ukw.de

1 Interdisciplinary Center, Palliative Medicine, University Hospital Würzburg, Josef-Schneider-Str. 11, B1, 97080 Würzburg, Germany

2 Department of Psychology (Biological Psychology, Clinical Psychology, and Psychotherapy), University of Würzburg, Würzburg, Germany

3 Center of Mental Health, University of Würzburg, Würzburg, Germany

4 Comprehensive Cancer Center Mainfranken, University Hospital Würzburg, Würzburg, Germany

\section{Introduction}

Detection and treatment of cancer have significantly improved, leading to increased survival time in cancer patients. However, contemporary treatment methods are not without side effects [1]. As a result, side effects of cancer and their treatment gain more and more attention. Fatigue, one of the most common side effects of cancer and cancer-related treatment, is described as intense and chronic tiredness on a physical, emotional, and cognitive level, which is not related to previous activities and cannot be entirely reduced by sleep [2]. Twenty to eighty percent of cancer patients suffer from fatigue during therapy [3], and fatigue can persist even 5 years after therapy completion [4]. Increased interleukins [2, 3], anemia, and psychological stress $[4,5]$ might play a significant causal role in the etiology of fatigue, but the complexity of this interaction is not 
yet fully understood. Therefore, symptom-oriented treatment of fatigue is usually preferred to cause-specific treatment. The National Comprehensive Care Network recommends yoga as a category 1/grade A non-pharmacologic intervention for cancer patients during and after cancer-related therapy. Yoga interventions of different styles and duration were effective in reducing fatigue in cancer patients [6-10]. Meta-analyses of yoga with cancer patients report small to moderate effects on fatigue [11-14].

Findings on the long-term effects of yoga in cancer patients are less coherent than those for short-time effects. Some randomized controlled trials report a significant reduction in fatigue after yoga therapy, but no effect after 3 months compared to control groups without interventions $[15,16]$. Another randomized controlled trial finds no significant improvement in fatigue directly after yoga compared to a control group, but 3 months later [17]. In an observational study without a control group and with pre-post measurements, yoga intervention also showed significant long-term effects on fatigue and on depression, and anxiety months after completing of the yoga intervention [18].

The sustainability of such positive effects of yoga interventions is related to patients' practice frequency during and after the intervention [17, 19]. Patients suffering from fatigue are physically, emotionally, and cognitively exhausted. Therefore, it is quite comprehensible that patients affected by fatigue have difficulties motivating themselves to practice independently outside of regular classes [20]. Knowledge about physical activity and its impact on quality of life, physical activity before diagnosis [20], social support, and a given structure and appointed times for sports classes [21] have a positive influence on the patients' motivation for physical activity. Clinical depression, in contrast, keeps patients from physical activity [20]. To facilitate patients' independent practice, it is an established method to provide them with an exercise $\mathrm{CD}$ and exercise book at the end of a yoga intervention [16, 22-24]. Individual documentation of the daily exercise duration in addition to the exercise $\mathrm{CD}$ was also proven to be helpful concerning the sustainability of positive effects [17]. Although these methods aim to establish regular yoga exercises, they are usually offered only once at the end of the yoga therapy, and due to a lack of regularity and frequency, they do not represent a reminder of the yoga practice.

Daily reminder e-mails help increase adherence to medication [25, 26]. Cicolini et al. (2014) also used weekly reminder e-mails to remind patients with cardiovascular risk factors to adopt a healthy lifestyle. This led to significantly lower cardiovascular risk factors compared to a group that did not receive reminder e-mails. However, there are no comparable studies on yoga for improving fatigue symptoms that use weekly reminder e-mails to promote lasting effects.
In a previous paper, we described the efficacy of yoga therapy regarding fatigue, depression, and quality of life. Patients who participated in an 8-week yoga therapy reported significantly lower general and physical fatigue and depression and higher quality of life compared to patients in a waiting list control group. A higher attendance rate was associated with more reduction in fatigue [10]. This paper builds on the results of the previous paper and examines the stabilization of the efficacy of yoga therapy through reminder e-mails as described above and the long-term effects of yoga therapy as an additional research question.

Thus, the first research question of this study should examine the efficacy of reminder e-mails on fatigue and its subscores compared to patients who do not receive reminder e-mails [27]. We hypothesized that patients who receive reminder e-mails would report lower fatigue scores. Our second research question aimed to examine the efficacy of reminder e-mails on increasing practice frequency compared to patients without reminder e-mails. The hypothesis was that patients with reminder e-mails practice more frequently than patients without reminder e-mails. The third hypothesis was that the effect of reminder e-mails on fatigue and its subscores would be mediated by practice frequency. The fourth research question addressed the long-term changes in self-reported fatigue, depression, and quality of life after an 8 -week yoga intervention for patients with different types of cancer after 6 months. We hypothesized that fatigue would be significantly lower immediately after yoga therapy and at the follow-up 6 months later compared to the baseline.

\section{Methods}

\section{Design and procedure}

This randomized controlled trial took part in the University Hospital Würzburg from November 2018 to September 2020. After informed consent, patients participated in 8-week yoga classes for $1 \mathrm{~h}$ a week. At the end of the yoga therapy, participants received a booklet and a CD with instructions of the asanas they learned in class. Afterward, participants were randomly assigned to "reminder" or "no-reminder" group. A block randomization procedure was used. Patients assigned to a yoga group form a block. The randomization list with computer-generated numbers was compiled by staff members of the Palliative Medicine Centre. The reminder group received a weekly reminder e-mail over 24 weeks. Primary (fatigue, practice frequency) and secondary outcomes (depression, quality of life) were assessed before and after yoga therapy and 24 weeks after the end of yoga therapy via self-report questionnaires. The Ethics Commission of the University of Würzburg has 
approved the study in advance (59/18-sc). The study design can be found in the protocol article [28].

\section{Participants}

Participants of this randomized controlled trial were adult cancer patients, at least 18 years old, who were planning to undergo cancer treatment in University Hospital Würzburg at the time of screening. In order to be included, patients had to report at least mild fatigue symptoms in the Fischer screening (intensity $\geq 1$, impairment $\geq 1$ ) [29]. Exclusion criteria were insufficient knowledge of the German language, severe emotional or physical impairment, and more than $50-\mathrm{km}$ distance to the university hospital.

\section{Yoga intervention}

The yoga intervention ran for 8 weeks. Patients took part in one session of $1 \mathrm{~h}$ per week. The yoga intervention consisted of different asanas (physical postures with awareness), small series of conscious breathing (pranayama), and deep relaxation (savasana) at the beginning and the end of each class, inspired by a mindfulness-based stress reduction program (MBSR) [30]. The exercises were structured from lying to sitting to standing and kept constant for all sessions. In all exercises, participants were reminded to breathe slowly, deeply, and consciously. Nonviolence (ahimsa), as an important basic principle of yoga, was repeated in every session and helped to encourage the participants to be gentle with their bodies and personal limitations.

\section{E-mail reminder}

After completing of yoga therapy, personal e-mails were sent on the same day every week, for 24 weeks altogether. In the first 12 weeks, e-mails contained descriptions of twelve yoga exercises-one exercise each week-and a personal encouragement to practice yoga independently during this week. The yoga exercises, known to the participants from the yoga classes, were described analogously to the description in the group yoga classes. In the following 12 weeks, the twelve reminder e-mails with these exercises were repeated in the same order.

\section{Questionnaires}

Outcomes were assessed directly before and after yoga therapy (T0 and T1) and 6 months later (T2).

\section{Primary outcomes}

Self-reported fatigue Self-reported fatigue was assessed using the German version of EORTC QLQ-FA13-13 items (European Organization for Research and Treatment of Cancer-Quality of Life Questionnaire-Fatigue) [31]. This questionnaire can be used in all tumor diseases in all stages and phases of the disease and all areas of treatment (chemotherapy, radiation, surgery) or care (acute care, rehabilitation, aftercare, or palliative care) [32]. The EORTC QLQ-FA13 measures general fatigue using 13 items overall. Physical fatigue was assessed with five items (e.g., have you felt exhausted?), emotional fatigue with three items (e.g., did you feel discouraged?), and cognitive fatigue with two items (e.g., did you have trouble thinking clearly?). Response categories of all items are "not at all," "a little," "quite a bit," and "very much," coded with scores from 1 to 4 . Mean scores are calculated for each subscale and the overall scale and then linearly transformed to symptom scales ranging from 0 to 100 . Higher values indicate a higher level of fatigue symptoms. The internal consistency for the German version was good, with Cronbach's alpha values ranging from 0.79 to 0.90 [31].

Practice frequency The practice frequency was assessed after yoga therapy and 6 months later with open responses. Patients indicated how many days they had practiced in an average week and how many minutes they practiced on average per practice day. The variable was calculated by multiplication of average days and average minutes.

\section{Secondary outcomes}

Depression and quality of life Depression was assessed using the Patient Health Questionnaire (PHQ-9), which assesses symptoms of depression according to the DSM-V [33]. Higher values indicate higher depression. The internal consistency for the PHQ-9 proved to be good with Cronbach's $\alpha=0.79$ for cancer patients [34].

Quality of life was assessed with the function scale of EORTC QLQ-C15-PAL [35]. This scale consists of the item How would you rate your overall quality of life during the past week?, scaled from 1 (very poor) to 7 (excellent). Higher scores represent a higher quality of life.

Sociodemographic and health data The following sociodemographic data was assessed at T0: age, gender, marital status, number of children, educational level, professional status, tumor diagnosis. In addition, personal benefits of yoga and reasons not to practice (anymore) were assessed at $\mathrm{T} 2$ on a four-point Likert scale (does not apply at all, applies completely).

\section{Statistical analyses}

All analyses were performed on intention-to-treat basis on a significance level of $\alpha=.0 .05$. Data analysis was carried out 
using IBM SPSS Statistics version 26. For the first hypothesis, analyses of variance (ANOVA) for fatigue and each subscale (physical, emotional, and cognitive) using time (T1 vs. T2) as within-factor and type of reminder (reminder vs. no-reminder) as a between-subject factor were performed. For the second hypothesis, we calculated a $t$ test for independent samples (reminder vs. no-reminder) with residual gain scores (RGS) of practice frequency [36] as the outcome variable. RGS were calculated by subtracting the standardized $z$ values of the practice frequency at T1 multiplied with the correlation of $\mathrm{T} 1$ and $\mathrm{T} 2$ from the standardized $z$ values of the practice frequency at T2. RGS control baseline differences and measurement errors occurring in the use of repeated measures on the same instrument [36]. The third research question was tested using a mediation model with group (reminder vs. no-reminder) as a predictor, RGS of practice frequency as a mediator, and RGS of fatigue and subscores as dependent variable. Mediation analyses were performed using the PROCESS macro by Hayes (2018) [37]. The 95\% confidence interval of the indirect effects was obtained with 5000 bootstrap resamples [38]. The fourth research question was tested with an ANOVA and paired $t$ tests with adjusted $\alpha=0.016$ to analyze long-term changes in fatigue, depression, and quality of life between baseline, after yoga, and follow-up.

\section{Results}

\section{Participant flow and assignment}

Patients were recruited between November 2018 and December 2019. One hundred seventy-two patients agreed to participate and were randomly assigned to a reminder $(n=85)$ or no-reminder group $(n=87)$. Due to dropouts and non-returned questionnaires, a total of 102 patients (reminder $n=51$, no-reminder $n=51$ ) were finally included in the analyses. For a detailed description of participant flow, see Fig. 1.

\section{Demographics and clinical characteristics}

Participants' age ranged from 24 to $82(M=59.2, \mathrm{SD}=11.5)$, $72.5 \%$ were female, and $68.8 \%$ were married or in a longterm relationship. The participants were predominantly diagnosed with breast cancer (52.5\%), followed by prostate cancer $(13.1 \%)$ and lymphoma (9.1\%). Eighty-six percent were under cancer-specific treatment at $\mathrm{T} 0$ (see Table 1). There were no significant differences between reminder and no-reminder groups in demographic (age, gender, marital status) or health-related (cancer, treatment state) characteristics.

\section{Practice frequency and evaluation of reminder e-mails}

Of the reminder group and no-reminder group, $74.5 \%$ and $62.0 \%$ reported 6 months after therapy that they were currently practicing yoga. Patients in the reminder group reported an average practice of $39.7 \mathrm{~min}$ per week at $\mathrm{T} 1$ $(\mathrm{SD}=44.7)$ and $68.5 \mathrm{~min}(\mathrm{SD}=57.0)$ at $\mathrm{T} 2$. In the noreminder group, the self-reported average practice time was $36.3 \mathrm{~min}$ at $\mathrm{T} 1(\mathrm{SD}=43.1)$ and $39.9 \mathrm{~min}(\mathrm{SD}=39.1)$ at $\mathrm{T} 2$. The main reason for not practicing yoga anymore in both groups was lacking motivation without professional instruction (reminder: $55.6 \%$, no-reminder: $58.6 \%$ ). For more perceived benefits of yoga and reasons not to practice (anymore), see Table 2.

In the reminder group, $74.0 \%$ reported having read the e-mails always or frequently, only $8.0 \%$ indicated they never or almost never read them, $67.3 \%$ reported that they were strongly or very strongly reminded of yoga therapy by the e-mails, and 54\% found the e-mails (very) helpful to keep up their independent yoga practice. Eighty-six percent of the patients felt that the e-mails were not at all or very little annoying.

\section{Primary outcome: efficacy of reminder e-mails on fatigue and yoga practice}

Fatigue The reminder group reported a significant reduction from T1 to T2 compared to the no-reminder group in general fatigue $(F(100 ; 1)=4.420 ; p=0.038 ; d=0.42)$ as well as in emotional fatigue $(F(99 ; 1)=8.538 ; p=0.004 ; d=0.59)$. No significant effects were found for physical and cognitive fatigue (see Table 3/Fig. 2). Controlling for baseline differences with analysis of covariance (ANCOVA), group (reminder vs. no-reminder) differed significantly in emotional fatigue $(F(98 ; 1)=6.43 ; p=0.013 ; d=0.51)$, but not in general fatigue $(F(99 ; 1)=3.611 ; p=0.06)$.

Practice frequency The mean of RGS of practice frequency score differed significantly between the reminder group $(m=0.28, \mathrm{SD}=1.14)$ and the no-reminder group $(m=-0.24, \mathrm{SD}=0.79)(t(86)=2.47, p=0.015,95 \% \mathrm{CI}$ $[-0.94 ;-0.10])$ with a medium effect size $d=0.52$.

Influence of practice frequency on fatigue A mediation analysis was conducted to test whether practice frequency mediates the effect of reminder e-mails on fatigue and its subscales [37-40]. Significant correlations between reminder e-mails, practice frequency, and fatigue could only be found for emotional fatigue $(r[0.28 ; 0.51] ; p<0.02)$, so the mediation model was tested only for emotional fatigue. First, reminder e-mails were positively associated with RGS of emotional fatigue $(B=-0.42,95 \%$ CI $[-0.79 ;-0.09]$, 


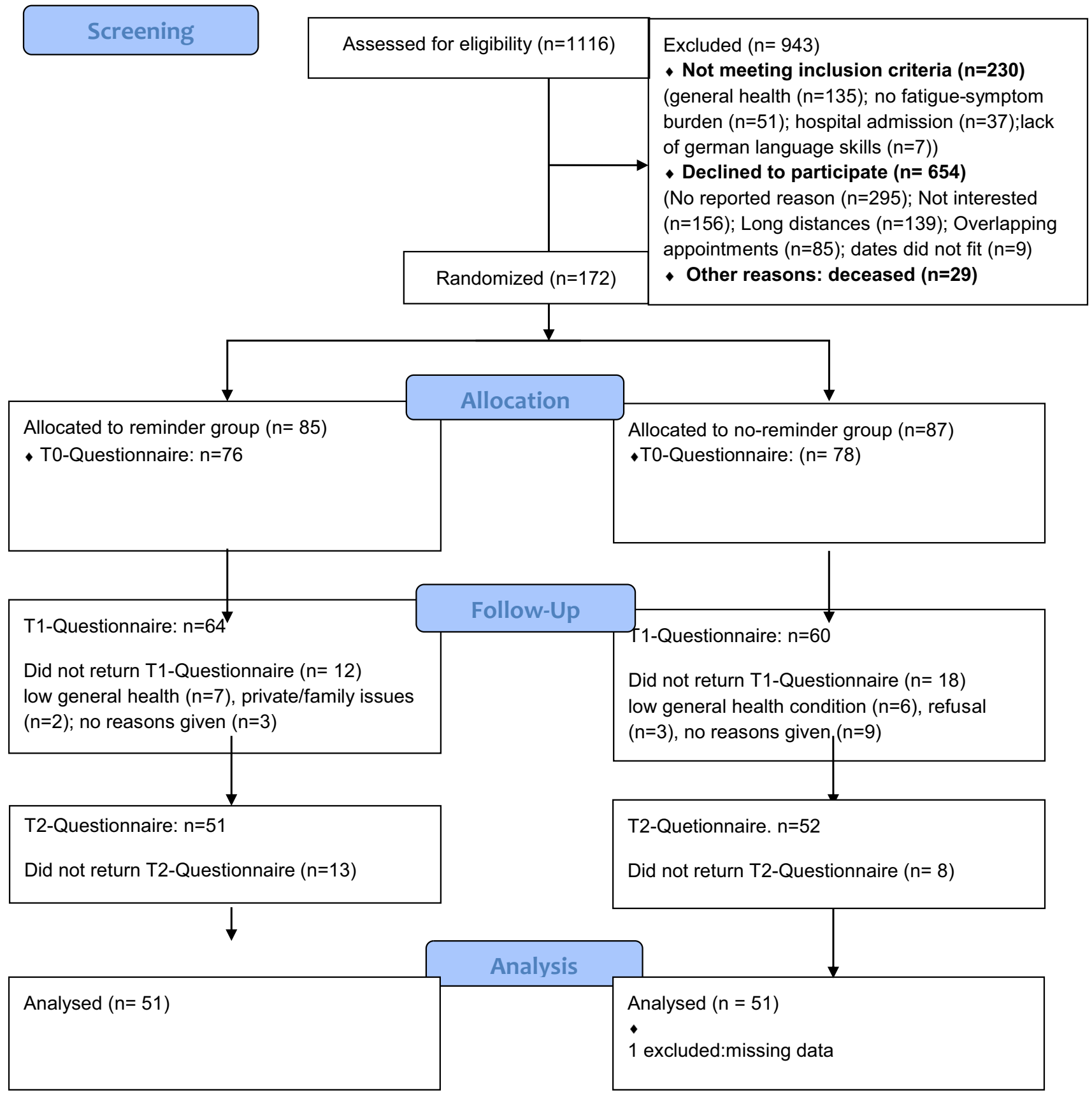

Fig. 1 CONSORT diagram showing screening, allocation, and participant flow by group

$p=0.013)$. Second, there was a significant relation between reminder e-mails and the mediator RGS of practice frequency $(B=0.52,95 \%$ CI $[0.10 ; 0.94], p=0.015)$. In a third step, the relation between practice frequency and emotional fatigue was not significant $(B=-0.18,95 \%$ CI $[-0.36 ; 0.01], p>0.05)$. The direct effect of e-mail on emotional fatigue mediated by practice frequency was lower $(B=-0.32,95 \%$ CI $[-0.69 ; 0.05], p=0.09)$ than the total effect $(B=-0.42)$. The indirect effect of e-mail on practice frequency and practice frequency on emotional fatigue was $B=-0.09,95 \%$ CI $[-0.22 ;-0.004]$.

\section{Secondary outcome: long-term effects}

The assumption of sphericity was not met. Therefore, all following ANOVAs were adjusted according to Greenhouse-Geisser. Regarding general, as well as physical, emotional, and cognitive fatigue, there were significant 
Table 1 Demographic and clinical characteristics of study population by group

\begin{tabular}{|c|c|c|c|}
\hline Characteristics & All $(N=102)$ & Reminder $(N=51)$ & No-reminder $(N=51)$ \\
\hline Age [mean (SD)] & $59.2(11.5)$ & $56.6(10.7)$ & 61.7 (11.7) \\
\hline \multirow[t]{2}{*}{ Range } & $24-82$ & $34-81$ & $36-82$ \\
\hline & $\%(N)$ & $\%(N)$ & $\%(N)$ \\
\hline Female & $72.5(74)$ & $78.4(40)$ & $66.7(34)$ \\
\hline \multicolumn{4}{|l|}{ Marital status } \\
\hline Married/partnered & $68.8(70)$ & $70.6(36)$ & $66.7(34)$ \\
\hline Never married/single & $11.8(12)$ & $7.8(4)$ & $15.7(8)$ \\
\hline Divorced/separated & $12.7(13)$ & $17.6(9)$ & $7.8(4)$ \\
\hline Widowed & $4.9(5)$ & $2.0(1)$ & $7.8(4)$ \\
\hline \multicolumn{4}{|l|}{ Education level } \\
\hline Primary education & $29.4(30)$ & $25.5(13)$ & $33.3(17)$ \\
\hline Secondary education & $27.5(28)$ & $25.5(13)$ & $29.4(15)$ \\
\hline Tertiary education & $40.2(41)$ & $47.0(24)$ & $33.3(17)$ \\
\hline Others & $1.0(1)$ & $0.0(0)$ & $2.0(1)$ \\
\hline \multicolumn{4}{|l|}{ Tumor diagnosis } \\
\hline Breast cancer & $52.5(52)$ & $60.4(29)$ & $45.1(23)$ \\
\hline Prostate cancer & $13.1(13)$ & $10.4(5)$ & $15.7(8)$ \\
\hline Gastrointestinal cancer & $7.1(7)$ & $10.4(5)$ & $3.9(2)$ \\
\hline Lung cancer & $5.1(5)$ & $0.0(0)$ & $9.8(5)$ \\
\hline Lymphoma & $9.1(9)$ & $8.3(4)$ & $9.8(5)$ \\
\hline Gynecological cancer & $6.1(6)$ & $6.3(3)$ & $5.9(3)$ \\
\hline Head and neck cancer & $3.0(3)$ & $0.0(0)$ & $5.9(3)$ \\
\hline Cancer of CNS & $2.0(2)$ & $2.0(1)$ & $2.0(1)$ \\
\hline Other cancer & $2.0(2)$ & $2.0(1)$ & $2.0(1)$ \\
\hline \multicolumn{4}{|l|}{ Metastases } \\
\hline Present & $18.6(19)$ & $15.7(8)$ & $21.6(11)$ \\
\hline \multicolumn{4}{|c|}{ Therapy status 6 months after yoga therapy } \\
\hline Had a cancer-related therapy & $44.1(45)$ & $58.8(30)$ & $29.4(15)$ \\
\hline Chemotherapy & $5.9(6)$ & $5.9(3)$ & $5.9(3)$ \\
\hline Radiation therapy & $1.0(1)$ & $2.0(1)$ & $0.0(0)$ \\
\hline Hormone therapy & $28.4(29)$ & $37.3(19)$ & $19.6(10)$ \\
\hline Antibody therapy & $7.8(8)$ & $5.9(3)$ & $9.8(5)$ \\
\hline Other & $10.8(11)$ & $17.6(9)$ & $3.9(2)$ \\
\hline
\end{tabular}

$S D$ standard deviation, $C N S$ central nervous system time effects $(F>7.46, p<0.001, d>0.54)$. In the subsequent paired $t$ tests with alpha adjustment, significant effects between T0 and T1 $(T>3.11, p<0.002)$ were found for fatigue and its subscores. This significant difference was also evident between baseline (T0) and 6 months after completion of yoga therapy (T2) $(T>3.10, p<0.002)$. Similar significant results were found for depression $(F(202 ; 1.80)=24.95 ; p<0.001)$ and quality of life $(F(186 ; 1.86)=15.58, p<0.001)$. Regarding the paired $t$ test, there were also significant differences between $\mathrm{T} 0$ and $\mathrm{T} 1(T>4.76, p<0.001)$ and $\mathrm{T} 0$ and $\mathrm{T} 2(T>4.48$, $p<0.001)$. Between T1 and T2, no significant differences were found on any of the scales $(p>0.35)$.

\section{Discussion}

This randomized controlled trial examined the efficacy of reminder e-mails on yoga practice and fatigue in oncological patients with different types of cancer. Yoga for reducing fatigue is well-evaluated in cancer patients, but findings on the long-term effects of yoga are rare [17, 18, $41,42]$. Even fewer studies considered practice frequency as a potential mediator [17].

To the best of our knowledge, there has not yet been any study that actively tried to enhance the efficacy of yoga therapy by sending reminder e-mails. Reminder e-mails 
Table 2 Practice frequency, subjective benefits of yoga, and reasons not to practice (higher values represent higher agreement range 1-4)

\begin{tabular}{lll}
\hline & Reminder group & No-reminder group \\
\hline Yoga practice frequency & $N=51$ & $N=51$ \\
Practiced yoga in the last 6 months $(\%(\mathrm{~N}))$ & $88.2(45)$ & $72.5(37)$ \\
Practiced yoga currently at the moment $(\%(\mathrm{~N}))$ & $74.5(38)$ & $62.0(31)$ \\
Average amount of yoga practice a week in minutes (m (SD)) & $68.5(56.9)$ & $39.9(39.0)$ \\
Subjective benefits of yoga (m(SD)) & $N=47$ & $N=44$ \\
Better concentration & $2.72(0.90)$ & $2.57(0.87)$ \\
Less tired & $2.77(0.84)$ & $2.65(0.84)$ \\
Feeling more fit & $3.47(0.78)$ & $3.27(0.79)$ \\
Better dealing with cancer disease & $2.98(0.87)$ & $3.02(0.92)$ \\
Better dealing with anxiety concerning my cancer disease & $2.94(0.90)$ & $2.73(0.90)$ \\
Less worried about my future & $2.89(0.91)$ & $2.81(0.88)$ \\
Less sad/depressed & $3.11(0.84)$ & $3.05(0.82)$ \\
Reasons to stop practicing yoga (m (SD)) & $N=18$ & $N=29$ \\
Not motivated without professional instruction & $2.11(1.4)$ & $2.66(1.0)$ \\
Physically not able to do yoga & $1.68(1.25)$ & $1.90(1.0)$ \\
No need for yoga due to good state of health & $1.24(0.75)$ & $1.82(0.77)$ \\
No benefit of yoga therapy & $1.44(1.11)$ & $1.30(0.61)$ \\
Subjective experienced harm through yoga & $1.12(0.93)$ & $1.21(0.49)$ \\
No time for yoga in everyday life & $2.06(1.56)$ & $1.89(0.89)$ \\
\hline
\end{tabular}

$S D$ standard deviation, $N A$ not applicable

Table 3 Means (m), standard deviation (SD), and $p$ values of ANOVA analyses of time and group effects, and time $\times$ group interaction between reminder and no-reminder group for fatigue and subscales

\begin{tabular}{|c|c|c|c|c|c|c|c|}
\hline & \multicolumn{2}{|l|}{ Reminder } & \multicolumn{2}{|l|}{ No-reminder } & \multirow{2}{*}{$\begin{array}{l}\text { Time } \\
p \text { value }\end{array}$} & \multirow{2}{*}{$\begin{array}{l}\text { Group } \\
p \text { value }\end{array}$} & \multirow{2}{*}{$\begin{array}{l}\text { Time } \times \text { group } \\
p \text { value }\end{array}$} \\
\hline & $\begin{array}{l}\mathrm{T} 1 \mathrm{~m}(\mathrm{SD}) \\
N=51\end{array}$ & $\begin{array}{l}\mathrm{T} 2 \mathrm{~m}(\mathrm{SD}) \\
N=51\end{array}$ & $\begin{array}{l}\mathrm{T} 1 \mathrm{~m}(\mathrm{SD}) \\
N=51\end{array}$ & $\begin{array}{l}\mathrm{T} 2 \mathrm{~m}(\mathrm{SD}) \\
N=51\end{array}$ & & & \\
\hline Fatigue & $33.97(20.7)$ & $30.45(21.49)$ & $30.78(16.02)$ & $33.98(18.47)$ & 0.919 & 0.962 & $0.038^{*}$ \\
\hline Physical fatigue & $45.23(24.30)$ & $42.48(25.12)$ & $42.80(20.21)$ & $44.40(20.98)$ & 0.779 & 0.825 & 0.289 \\
\hline Emotional fatigue & $28.89(26.47)$ & $22.22(29.09)$ & $21.78(22.32)$ & $30.06(28.40)$ & 0.753 & 0.937 & $0.004 *$ \\
\hline Cognitive fatigue & $18.62(21.25)$ & $16.01(16.65)$ & $20.26(13.46)$ & $20.92(20.51)$ & 0.610 & 0.288 & 0.396 \\
\hline
\end{tabular}

have been used predominantly in the medical context to improve compliance and adherence to appointments [25, $26,43]$, but not yet regarding such a complex construct with physical, emotional, and cognitive aspects as fatigue. We found that patients who received reminder e-mails had significantly lower general and emotional fatigue scores 6 months after the end of yoga therapy with small to medium effect sizes $(d=0.42-0.59)$ compared to the group who did not receive reminder e-mails. However, we did not find significant effects regarding physical and cognitive fatigue. Furthermore, the reminder group reported a significantly higher increase in practice frequency than the noreminder group. The mediation analysis also showed a significant relation between reminder e-mails and an increase in practice frequency. The reduction in emotional fatigue observed in this study due to reminder e-mails was partially mediated by practice frequency. Practice frequency at home may explain, at least in part, the association of reminder e-mails and emotional fatigue. However, there are other factors like mindfulness or therapy status that elucidate further variance (Fig. 3).

In several studies, higher attendance rates in yoga classes or higher practice frequency during and after yoga therapy significantly reduced fatigue $[10,17,44]$. In the current study, this could be confirmed only for emotional fatigue and general fatigue. Although reminder e-mails lead to a significantly higher practice frequency and patients in the reminder group reported lower general and emotional fatigue, a higher practice frequency was not associated with lower physical fatigue symptoms, as it was in other studies $[10,17]$. Patients in the reminder group received weekly specific yoga instructions. Therefore, they may have felt more supported and less alone by the personal e-mails than the group without reminder e-mails. This may, for example, have 
Fig. 2 Mean changes in $\mathbf{A}$ EORTC QLQ-FA12 general fatigue and B EORTC QLQFA12 emotional fatigue between $\mathrm{T} 1$ and $\mathrm{T} 2$ in e-mail group and no-e-mail group. Results show means and $95 \% \mathrm{CI}$

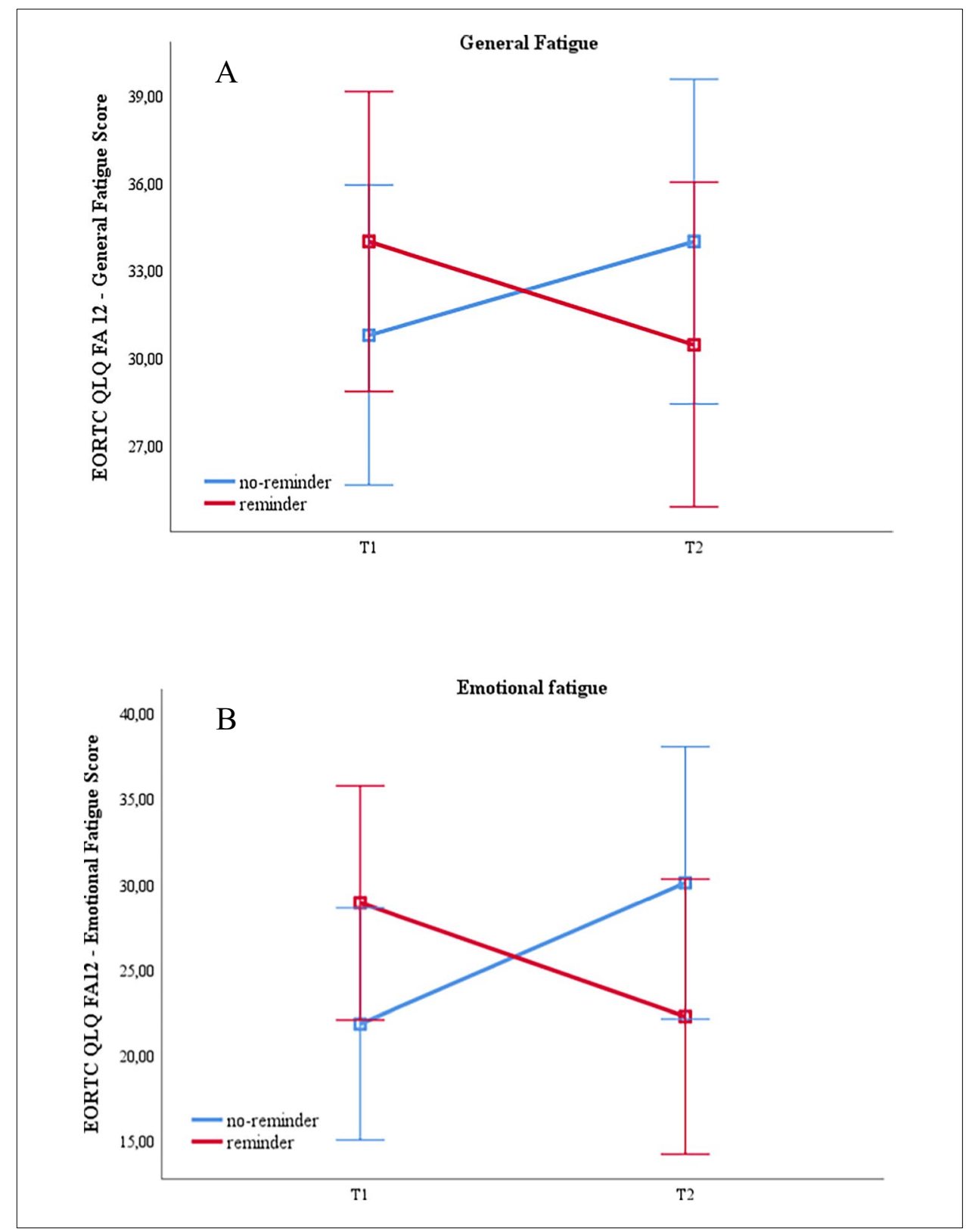

increased their self-efficacy [44] and motivation to do yoga, which might reflect in the increased practice frequency. By actively working to improve symptoms while receiving support through reminder e-mails, they might have felt less discouraged, helpless, and frustrated than the no-reminder group, leading to an improvement of emotional fatigue.

The independent yoga exercise at home was only recorded quantitatively but not qualitatively. For successful yoga practice, mindful practice of the exercises is an important basis [30]. In the study classes, the focus was set on mindful practice through the yoga teacher's instructions, and a higher attendance rate led to more improvement in fatigue [10]. However, this level of mindfulness in yoga practice cannot be assumed during the practice at home due to a potentially higher prevalence of disturbance factors and patients' comparatively little expertise in mindfulness. A lack of mindfulness in performing the exercises at home could be why there was no improvement in physical fatigue. Further research is needed to test the influence of mindfulness on the efficacy of home-based yoga. In addition, it must also be critically mentioned that the exercise time during the last 6 months was asked retrospectively. This might have led to a strong retrospective bias or social desirability issues. The tendency to answer in a socially desirable way might be higher for those who received personal e-mails for 24 weeks. Thus, future research may follow up on the present results by repeatedly assessing the quality and quantity of yoga exercises during the follow-up period instead of only once at the end. 


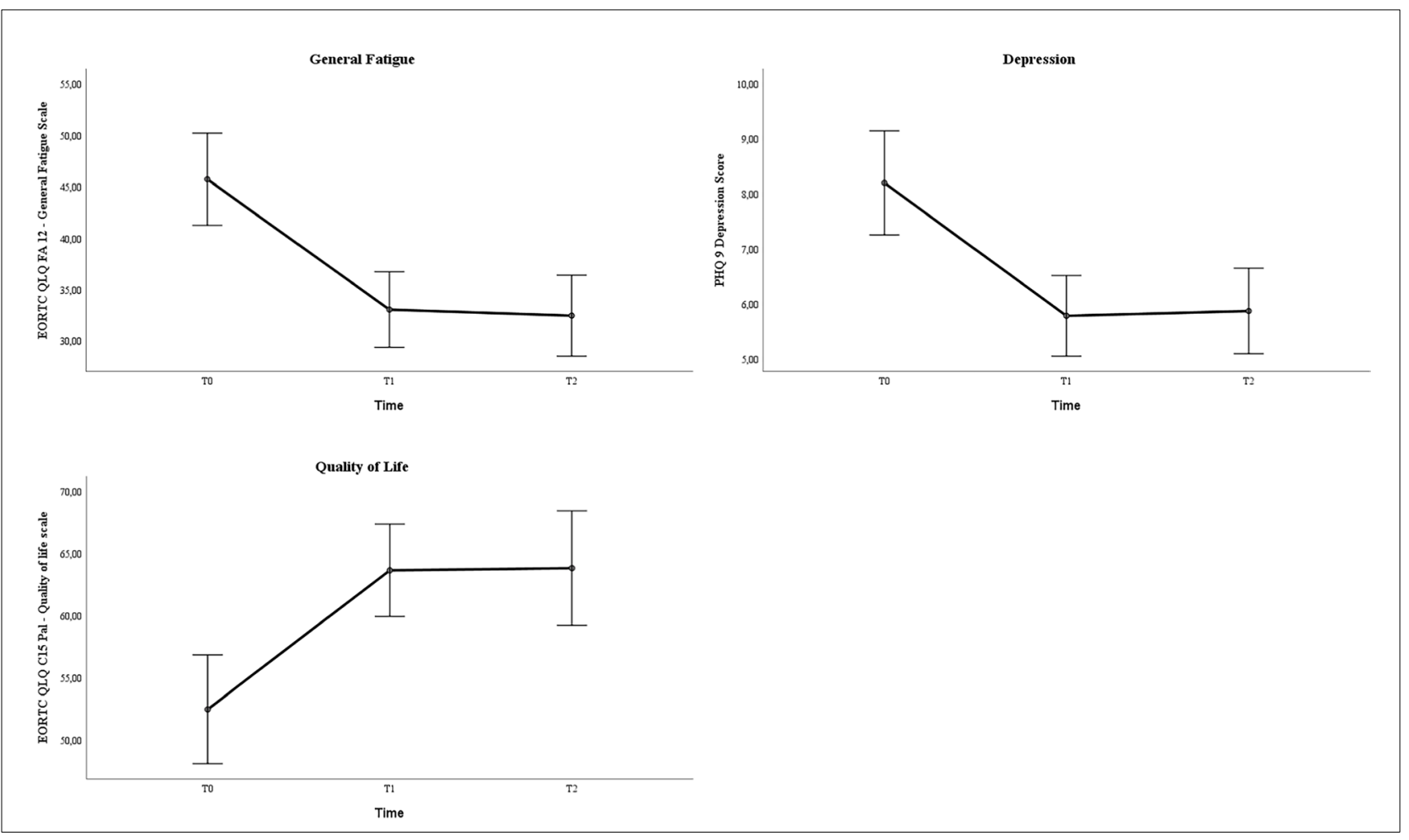

Fig. 3 Mean changes in A EORTC QLQ-FA12 general fatigue, B PHQ-9 Depression Score, and C EORTC QLQ-C15-PAL quality of life between T0, T1, and T2 in all participants. Results show means and 95\% CI

Although yoga is helpful in the beginning to reduce fatigue, it does not result in complete remission of fatigue symptoms. This is also reflected in the long-term results of this study. We found long-term improvements in fatigue, depression, and quality of life for all patients compared to baseline. Yoga can help reducing fatigue and depression and improving quality of life. These positive effects remained 6 months after the end of yoga therapy. However, there was no significant improvement between $\mathrm{T} 1$ and $\mathrm{T} 2$ after the end of yoga therapy. Yoga is helpful, especially during active cancer treatment [45], to a limited extent to reduce fatigue. Of the study population, $44.4 \%$ were still in therapy 6 months after yoga therapy. Only $40 \%$ of the participants reported a stable state of health at $\mathrm{T} 2$. Therefore, it is reasonable that both health status and continued therapy have a strong influence on the fatigue symptoms and that yoga can prevent further deterioration of the symptoms.

However, it must be critically considered that we did not include a control group that did not participate in yoga classes. Thus, the long-term improvement might not be solely due to yoga. Other factors, such as spontaneous remission, tumor treatment, and disease, alternative supportive offers, or social support, may have contributed to the improvement. Since most yoga studies observe brief followup periods from 6 to 12 weeks $[16,41,42]$, we decided to use a comparatively long follow-up period of 6 months. As a result, it would not have been ethically justifiable to place these patients on a 6-month waiting list because of cancer patients' short life expectancy. In addition to ethical reasons, the risk of low compliance with a very long waiting period also spoke against a waiting control group. Despite a high dropout between $\mathrm{T} 0$ and $\mathrm{T} 2$, of a total of $40 \%$ of the patients who agreed to the study initially, the response rate for the long follow-up of 6 months was very high at $83 \%$ compared to other studies.

In summary, previous studies supported that yoga helps reduce fatigue and that more participation in yoga leads to a higher reduction of symptoms [10]. However, our results suggest that more frequent practice does not lead to further reduction of fatigue after the end of yoga therapy but might help prevent fatigue symptoms from increasing again despite active cancer treatment. Furthermore, we have found positive effects of reminder e-mails on general and emotional fatigue after yoga therapy compared to a group without reminder e-mails. Reminder e-mails are feasible, very well accepted, and positively evaluated by cancer patients. In the long term, yoga can also help reduce fatigue and depression and improve the quality of life. Home-based yoga therapy with weekly reminder e-mails and explicit instructions for a mindful practice could be a combination that reduces the 
barrier of long journeys to join a yoga class in a yoga studio and increases personal motivation to individual practice.

Abbreviations CI: Confidence interval; EORTC QLQ-FA13: European Organization for Research and Treatment of Cancer-Quality of Life Questionnaire-Fatigue; MBSR: Mindfulness-based stress reduction; PHQ-9: Patient Health Questionnaire; QoL: Quality of life; RGS: Residual gain score

Authors' contribution vOB initiated the study and obtained funding. vOB, EJ, and TZ developed the study design. EJ developed the yoga session and material. AP participates in developing the design and statistical analysis plan. TZ and AR supervised the study and data collection. TZ wrote the first draft of the manuscript, which was critically reviewed by AP and AR. All authors finally read and approved the manuscript for submission.

Funding Open Access funding enabled and organized by Projekt DEAL. This work is supported by the German Cancer Aid, Buschstr., 32, 53113 Bonn, Germany. Funding covers costs for personal, materials, and traveling expenses. As a sponsor, German Cancer Aid plays no role in the study design, collection, analysis, interpretation, or writing of the manuscript. German Cancer Aid has peer-reviewed the study protocol.

\section{Declarations}

Ethics approval and consent to participate The investigation conforms to the principles outlined in the Declaration of Helsinki. The study protocol was approved by the Ethics Committee of the University Würzburg on 15/05/2018 (Nr. 59/18 sc). Eligible patients received written information about all relevant aspects of the study and that their participation in the study is voluntary and they have the right to refuse or to withdraw their consent at any time without reprisals. Patients provided written informed consent. The study has been registered with the German Clinical Trials Register DRKS00016034 and Clinical Trials.gov NCT04433793.

Role of the funding sources The design, conduct, data collection, analysis, and interpretation of the results were performed independently of the funders. The funders played no role in the review or approval of this manuscript.

Conflict of interest Zetzl and Renner report grants from Deutsche Krebshilfe/German Cancer Aid, during conduct of the study. The authors declare no competing interests.

Open Access This article is licensed under a Creative Commons Attribution 4.0 International License, which permits use, sharing, adaptation, distribution and reproduction in any medium or format, as long as you give appropriate credit to the original author(s) and the source, provide a link to the Creative Commons licence, and indicate if changes were made. The images or other third party material in this article are included in the article's Creative Commons licence, unless indicated otherwise in a credit line to the material. If material is not included in the article's Creative Commons licence and your intended use is not permitted by statutory regulation or exceeds the permitted use, you will need to obtain permission directly from the copyright holder. To view a copy of this licence, visit http://creativecommons.org/licenses/by/4.0/.

\section{References}

1. Allemani C, Matsuda T, Di Carlo V et al (2018) Global surveillance of trends in cancer survival 2000-14 (CONCORD-3). Lancet (London, England) 391(10125):1023-1075. https://doi.org/10. 1016/S0140-6736(17)33326-3

2. Bower JE (2007) Cancer-related fatigue. Brain Behav Immun 21(7):863-871. https://doi.org/10.1016/j.bbi.2007.03.013

3. Miller AH, Ancoli-Israel S, Bower JE et al (2008) Neuroendocrine-immune mechanisms of behavioral comorbidities in patients with cancer. J Clin Oncol 26(6):971-982. https://doi.org/10.1200/ JCO.2007.10.7805

4. Servaes P, Verhagen C, Bleijenberg G (2002) Fatigue in cancer patients during and after treatment. Eur J Cancer (Oxford, England: 1990) 38(1):27-43. https://doi.org/10.1016/s09598049(01)00332-x

5. Kuhnt S, Friedrich M, Schulte T et al (2019) Predictors of fatigue in cancer patients: a longitudinal study. Support Care Cancer 27(9):3463-3471. https://doi.org/10.1007/ s00520-019-4660-4

6. Banasik J, Williams H, Haberman M et al (2011) Effect of Iyengar yoga practice on fatigue and diurnal salivary cortisol concentration in breast cancer survivors. J Am Acad Nurse Pract 23(3): 135142. https://doi.org/10.1111/j.1745-7599.2010.00573.x

7. Bower JE, Greendale G, Crosswell AD et al (2014) Yoga reduces inflammatory signaling in fatigued breast cancer survivors. Psychoneuroendocrinology 43:20-29. https://doi.org/10.1016/j.psyne uen.2014.01.019

8. Carson JW, Carson KM, Olsen MK et al (2017) Mindful Yoga for women with metastatic breast cancer. BMC Complement Altern Med 17(1):153. https://doi.org/10.1186/s12906-017-1672-9

9. Cramer LR, Langhorst $J$ et al (2016) Is one yoga style better than another? A systematic review of associations of yoga style and conclusions in randomized yoga trials. Complement Ther Med 25:178-187. https://doi.org/10.1016/j.ctim.2016.02.015

10. Zetzl T, Renner A, Pittig A et al (2020) Yoga effectively reduces fatigue and symptoms of depression in patients with different types of cancer. Support Care Cancer. https://doi.org/10.1007/ s00520-020-05794-2

11. Buffart LM, van Uffelen JGZ, Riphagen II et al (2012) Physical and psychosocial benefits of yoga in cancer patients and survivors, a systematic review and meta-analysis of randomized controlled trials. BMC Cancer 12(1):559. https://doi.org/10.1186/ 1471-2407-12-559

12. Cramer $\mathrm{H}$, Lange $\mathrm{S}$, Klose $\mathrm{P}$ et al (2011) Can yoga improve fatigue in breast cancer patients? A systematic review. Acta oncologica (Stockholm, Sweden) 51:559-560. https://doi.org/10.3109/02841 86X.2011.637960

13. Danhauer SC, Addington EL, Cohen L et al (2019) Yoga for symptom management in oncology: a review of the evidence base and future directions for research. Cancer 125(12):1979-1989. https://doi.org/10.1002/cncr.31979

14. Dong B, Xie C, Jing X et al (2019) Yoga has a solid effect on cancer-related fatigue in patients with breast cancer: a meta-analysis. Breast Cancer Res Treat 177(1):5-16. https://doi.org/10.1007/ s10549-019-05278-w

15. Cramer H, Rabsilber S, Lauche R et al (2015) Yoga and meditation for menopausal symptoms in breast cancer survivors-a randomized controlled trial. Cancer 121(13):2175-2184

16. Bower JE, Crosswell AD, Stanton AL et al (2015) Mindfulness meditation for younger breast cancer survivors: a randomized controlled trial. Cancer 121(8):1231-1240. https://doi.org/10.1002/ cncr.29194

17. Kiecolt-Glaser JK, Bennett JM, Andridge R et al (2014) Yoga's impact on inflammation, mood, and fatigue in breast cancer 
survivors. J Clin Oncol 32(10):1040-1049. https://doi.org/10. 1200/JCO.2013.51.8860

18. Lundt A, Jentschke E (2019) Long-term changes of symptoms of anxiety, depression, and fatigue in cancer patients 6 months after the end of yoga therapy. Integr Cancer Ther 18:1534735418822096. https://doi.org/10.1177/1534735418 822096

19. Amritanshu RR, Rao RM, Nagaratna R et al (2017) Effect of longterm yoga practice on psychological outcomes in breast cancer survivors. Indian J Palliat Care 23(3):231-236. https://doi.org/ 10.4103/IJPC.IJPC_93_17

20. Frikkel J, Götte M, Beckmann M et al (2020) Fatigue, barriers to physical activity and predictors for motivation to exercise in advanced cancer patients. BMC Palliat Care 19(1):43. https://doi. org/10.1186/s12904-020-00542-Z

21. Mikkelsen MK, Nielsen DL, Vinther A et al (2019) Attitudes towards physical activity and exercise in older patients with advanced cancer during oncological treatment - a qualitative interview study. Eur J Oncol Nurs 41:16-23. https://doi.org/10. 1016/j.ejon.2019.04.005

22. Moadel AB, Shah C, Wylie-Rosett J et al (2007) Randomized controlled trial of yoga among a multiethnic sample of breast cancer patients: effects on quality of life. J Clin Oncol 25(28):4387-4395. https://doi.org/10.1200/JCO.2006.06.6027

23. Chandwani KD, Thornton B, Perkins GH et al (2010) Yoga improves quality of life and benefit finding in women undergoing radiotherapy for breast cancer. J Soc Integr Oncol 8(2):43-55

24. Vadiraja HS, Rao RM, Nagarathna R et al (2017) Effects of yoga in managing fatigue in breast cancer patients. Indian J Palliat Care 23(3):247-252. https://doi.org/10.4103/IJPC.IJPC_95_17

25. Fox MC, Creinin MD, Murthy AS et al (2003) Feasibility study of the use of a daily electronic mail reminder to improve oral contraceptive compliance. Contraception 68(5):365-371. https:// doi.org/10.1016/j.contraception.2003.08.013

26. Haramiova Z, Stasko M, Hulin M et al (2017) The effectiveness of daily SMS reminders in pharmaceutical care of older adults on improving patients' adherence to antihypertensive medication (SPPA): study protocol for a randomized controlled trial. Trials 18(1):334. https://doi.org/10.1186/s13063-017-2063-8

27. Berger AM, Wielgus K, Hertzog M et al (2010) Patterns of circadian activity rhythms and their relationships with fatigue and anxiety/depression in women treated with breast cancer adjuvant chemotherapy. Support Care Cancer 18(1):105-114. https://doi. org/10.1007/s00520-009-0636-0

28. Zetzl T, Schuler M, Renner A et al (2019) Yoga intervention and reminder e-mails for reducing cancer-related fatigue - a study protocol of a randomized controlled trial. BMC Psychol 7(1):64. https://doi.org/10.1186/s40359-019-0339-3

29. Fischer I, Weis J, Rüffer U et al. (2017) Cancer-related fatigue in the palliative situation. Z Palliativmed (18): 97-110

30. Kabat-Zinn J (2015) Die MBSR Yogaübungen: Stressbewältigung durch Achtsamkeit, 4th edn. Arbor Verlag, Freiburg im Breisgau

31. Weis J, Tomaszewski KA, Hammerlid E et al. (2017) International Psychometric Validation of an EORTC Quality of Life Module Measuring Cancer Related Fatigue (EORTC QLQ-FA12). J Natl Cancer Inst 109(5). https://doi.org/10.1093/jnci/djw273
32. Weis J (2013) Diagnostik und Erfassung der tumorassoziierten Fatigue. Forum 28(1):39-42

33. Löwe B, Kroenke K, Herzog W et al (2004) Measuring depression outcome with a brief self-report instrument: sensitivity to change of the Patient Health Questionnaire (PHQ-9). J Affect Disord 81(1):61-66. https://doi.org/10.1016/S0165-0327(03)00198-8

34. Hartung TJ, Friedrich M, Johansen C et al (2017) The Hospital Anxiety and Depression Scale (HADS) and the 9-item Patient Health Questionnaire (PHQ-9) as screening instruments for depression in patients with cancer. Cancer 123(21):4236-4243

35. Groenvold M, Petersen MA, Aaronson NK et al (2006) The development of the EORTC QLQ-C15-PAL. Eur J Cancer 42(1):55-64

36. Steketee G, Chambless DL (1992) Methodological issues in prediction of treatment outcome. Clin Psychol Rev 12(4):387-400. https://doi.org/10.1016/0272-7358(92)90123-P

37. Hayes AF (2018) Partial, conditional, and moderated moderated mediation: quantification, inference, and interpretation. Commun Monogr 85(1):4-40. https://doi.org/10.1080/03637751.2017. 1352100

38. Preacher KJ, Hayes AF (2008) Asymptotic and resampling strategies for assessing and comparing indirect effects in multiple mediator models. Behav Res Methods 40(3):879-891. https:// doi.org/10.3758/BRM.40.3.879

39. Baron RM, Kenny DA (1986) The moderator-mediator variable distinction in social psychological research: conceptual, strategic, and statistical considerations. J Pers Soc Psychol 51(6):1173

40. Rucker DD, Preacher KJ, Tormala ZL et al (2011) Mediation analysis in social psychology: current practices and new recommendations. Soc Pers Psychol Compass 5(6):359-371. https://doi. org/10.1111/j.1751-9004.2011.00355.x

41. Bower JE, Garet D, Sternlieb B et al (2012) Yoga for persistent fatigue in breast cancer survivors: a randomized controlled trial. Cancer 118(15):3766-3775. https://doi.org/10.1002/cncr.26702

42. Cramer H, Rabsilber S, Lauche R et al (2015) Yoga and meditation for menopausal symptoms in breast cancer survivors-a randomized controlled trial. Cancer 121(13):2175-2184. https:// doi.org/10.1002/cncr.29330

43. Liu Q, Abba K, Alejandria MM et al. (2014) Reminder systems to improve patient adherence to tuberculosis clinic appointments for diagnosis and treatment. Cochrane Database Syst Rev 2014(11): CD006594. https://doi.org/10.1002/14651858.CD006594.pub3

44. Buffart LM, Ros WJG, Chinapaw MJM et al (2014) Mediators of physical exercise for improvement in cancer survivors' quality of life. Psychooncology 23(3):330-338. https://doi.org/10.1002/pon. 3428

45. Lin P-J, Peppone LJ, Janelsins MC et al (2018) Yoga for the management of cancer treatment-related toxicities. Curr Oncol Rep 20(1):5. https://doi.org/10.1007/s11912-018-0657-2

Publisher's note Springer Nature remains neutral with regard to jurisdictional claims in published maps and institutional affiliations. 